\title{
THE DESIGN OF AN INTER-DIGITAL STRUCTURE FOR TWTs
}

\author{
V. Latha Christie, Lalit Kumar \\ Microwave Tube Research \& Development Centre, BE Complex, Jalahalli, Bangalore 560013, India \\ and \\ N. Balakrishnan \\ Department of Aerospace Engineering, Indian Institute of Science, Bangalore 560012, India
}

\begin{abstract}
A design procedure for an inter-digital slowwave structure for TWTs is presented using equivalent circuit approach. The results are refined using numerical simulations by modeling the structure using the software package MAFIA. The dimensions of the inter-digital structure are compared with those of a conventional coupled-cavity slow-wave structure designed at the same center frequency. It is concluded that the size and weight of the inter-digital structure is significantly reduced as compared to that of a coupledcavity structure.
\end{abstract}

\section{INTRODUCTION}

An inter-digital structure shown in Fig. I(a) is called so as it looks like fingers interlaced between cavities. It is an inverted slot mode (ISM) coupled-cavity slow-wave structure (CC-SWS) in which the slot is so large, that it operates in the slot pass-band [1],[2]. The conventional CC-SWS structure shown in Fig. 1(b), operates in the cavity passhand. The special features of ISM circuit are smaller cavity size and vanishing interaction impedance at the upper cutoff, which provides freedom from bandedge oscillations normally occurring in CC-SWS. However, the smaller interaction impedance at high frequencies leads to a steep fall in the gain with frequencies and to achieve a higher gain, the ISM circuit had to be made longer. The smaller cavity radius allows the cavity partition walls to be made of copper and the magnetic ferrule hole can be made to coincide with the cavity wall instead of the beam hole, which improves the thermal and focusing design of the TWT. Thus the magnetic focusing design can be decoupled from the slow-wave structure design.

A design procedure to obtain the dispersion and interaction impedance for an inter-digital structure, using equivalent circuit approach and numerical simulation is presented. A conventional coupled-cavity structure and an ISM structure were designed for the same center frequency, beam voltage and bandwidth. The dimension of the ISM structure were compared with those of the CC-SWS structure, and it was found that the cavity radius of ISM circuit is less than half of coupled-cavity structure and hence the size and weight of the tube can be reduced.
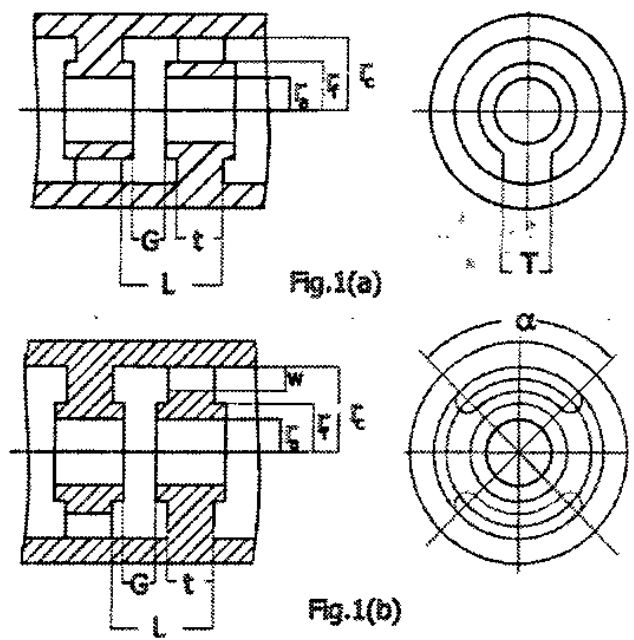

Fa.1(b)

Fig. 1. Schematic of an (a) Inter-digital circuit (b) Coupled cavity circuit

\section{DESIGN PROCEDURE}

The procedure for designing the ISM structure involves determination of cavity radius, finger width, shim thickness, cavity pitch, gap length, tunnel and ferrule radius. The cavity radius is determined by assuming the cavity as a foreshortened radial line resonator, but the cavity resonant frequency is chosen to be at the $\pi$ point of the second pass-band. The finger thickness largely determines the bandwidth of the lower pass-band. The reduction in finger thickness (the increase in coupling iris angle in CC-SWS) along with the reduction in cavity radius maintains the center operating frequency. As the cavity radius and hence the cavity height is small, the shim thickness can be chosen high, so that magnet saturation problem can be avoided and thermal dissipation can be improved. In the determination of the cavity pitch, the phase shiff/cavity is chosen near to $1.1 \pi$ due to the higher interaction impedance near the lower cutoff for an ISM circuit. And the beam velocity to phase velocity ratio is chosen higher than CCSWS due to the increase in the number of cavities required to achieve the desired gain. The gap length is chosen for maximum interaction impedance based on gap to pitch ratio. The bean-filling factor determines the tunnel radius and the concentration of electric field at the gap and the mechanical strength decides the ferrule radius. 


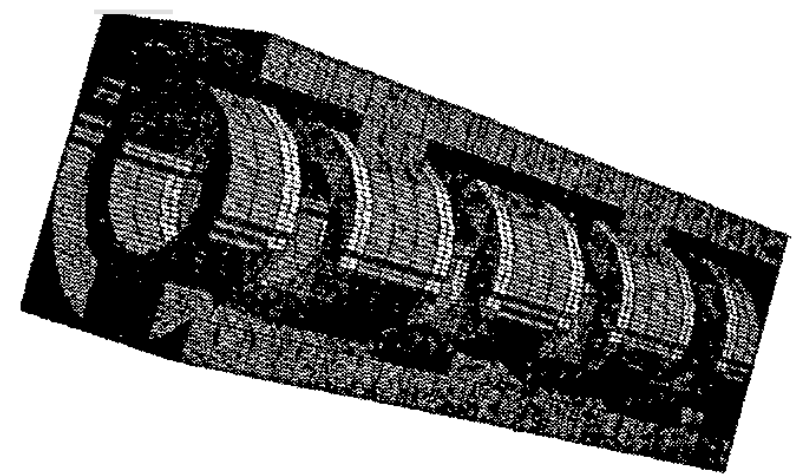

Fig. 2. 3-D model of inter-digital circuit.

After obtaining the cavity dimensions, the dispersion and interaction impedance are initially computed by equivalent circuit approach. The equivalent circuit procedure developed is similar to CCSWS [3] with some modifications in the calculation of slot capacitance and inductance, and Curnow's [4] equations for the computations of dispersion and impedance for coupling coefficient greater than half $(\mathrm{k}>1 / 2)$ is used. The result is also verified and finalized by numerical simulations by modeling the structure using the software package MAFIA. Eight cavities are modeled and nine resonant frequencies of the structure corresponding to the phase shifts of $\pi$ to $2 \pi$ at an interval of $0.125 \pi$ are obtained. Fig. 2 shows a 3-D cut away of four cavities of ISM circuit. For the computations of the interaction impedance, the group velocity is obtained by differentiating the dispersion characteristics at each frequency point and the energy and the electric field is obtained from the post processor section of MAFIA. The electric field is then integrated over the beam radius and then is Fourier analyzed and the value corresponding to the first space harmonic is found [5].

\section{RESULTS AND DISCUSSION:}

To compare the dimensions of the ISM structure with that of the CCSWS structure, both these structures are designed for the same center frequency, same beam

\begin{tabular}{|ll|l|l|}
\hline \multicolumn{2}{|l|}{ Normalized dimension } & \multicolumn{1}{|c|}{ ISM } & \multicolumn{1}{c|}{ CCSWS } \\
\hline Cavity Period & $\mathrm{L}$ & 0.1288 & 0.1625 \\
\hline Gap Length & $\mathrm{G}$ & 0.0383 & 0.0429 \\
\hline Tunnel Radius & $\mathrm{r}_{\mathrm{a}}$ & 0.0414 & 0.0414 \\
\hline Ferrule Radius & $\mathrm{r}_{\mathrm{f}}$ & 0.0613 & 0.0613 \\
\hline Slot Angle & $\alpha$ & $313^{\circ}$ & $105^{\circ}$ \\
\hline Slot Width & $\mathrm{w}$ & 0.0245 & 0.1288 \\
\hline Shim thickness & $\mathrm{t}$ & 0.0583 & 0.0583 \\
\hline Cavity radius & $\mathrm{r}_{\mathrm{c}}$ & 0.092 & 0.23 \\
\hline
\end{tabular}

Table 1 Normalized dimension of CCTWT and ISM

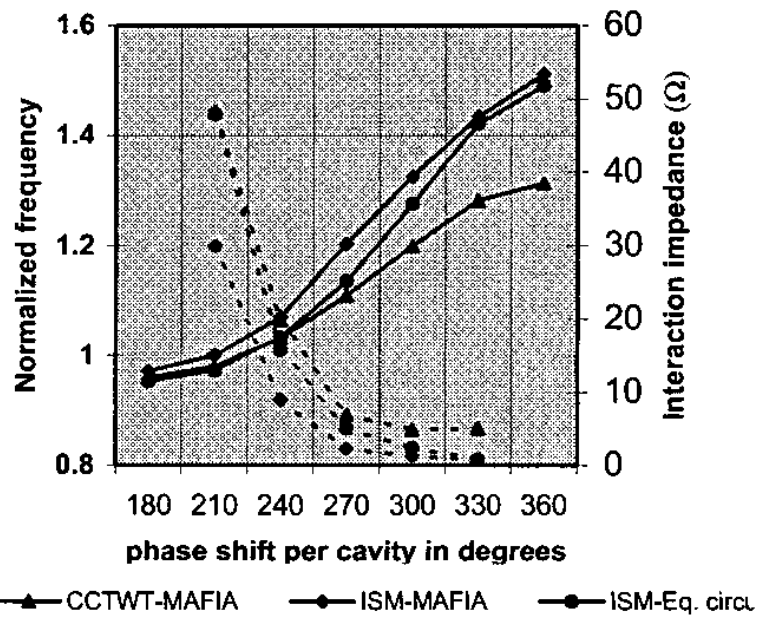

Fig. 3. Comparison of the dispersion and interaction impedance of CCTWT and ISM

voltage of $21 \mathrm{KV}$ and a bandwidth of $400 \mathrm{MHz}$ and the dimensions are given in table 1. The dimensions are normalized with respect to wavelength. The slot angle is deduced from the finger thickness ' $T$ ' for ISM circuit. The cavity radius of ISM circuit is less than half of its counterpart and hence the size and weight of the tube can be reduced. Fig. 3 gives the dispersion and interaction impedance characteristics for CCSWS and inter-digital structure, both from MAFIA and equivalent circuit approach. The frequencies are normalized with respect to the center frequency.

\section{ACKNOWLEDGEMENT}

The authors are thankful to the Director MTRDC, for his encouragement and permission to submit this work.

\section{REFERENCES}

1. J.R. Frey and I. Tammaru, A coupled-cavity TWT operating in an inverted slot mode, IEDM 1987, pp. 504-506.

2. Lebedev, Microwave Electronics, Moscow1974.

3. R.G Carter and Liu shunkang, Method for calculating the properties of coupled-cavity slow-wave structures from their dimensions, $\mathrm{P}$. IEE, Pt. H, 5, 1986, pp. 330-334.

4. H. J. Curnow, A generalized equivalent circuits for coupled cavity slow-wave structures, IEEE Trans. MTT-13, 5, 1965, pp. 671-675.

5. C. L. Cory and J.D. Wilson, 3-D simulation of traveling wave tube cold test characteristics using MAFIA, NASA TP- 3513, 1995. 